\title{
WARING PROBLEM WITH FACTORIALS
}

\author{
Moubariz Z. Garaev, Florian Luca and Igor E. ShParlinski
}

We show that any residue class $\lambda$ modulo $p$ can be represented in the form $n_{1} !+\cdots+$ $n_{\ell} ! \equiv \lambda(\bmod p)$ with $\ell=O\left((\log p)^{3} \log \log p\right)$.

\section{INTRODUCTION}

Let $p$ be an odd prime. Here, we continue to study exponential sums and various congruences with factorials which have been considered in $[1,2,3,5,6,7,8]$ and use some results and methods of $[2,3]$ to study the analogue of the Waring problem with factorials.

Namely, let $\ell(p)$ be the smallest integer $\ell \geqslant 1$ such that for every integer $\lambda$ the congruence

$$
n_{1} !+\cdots+n_{\ell} ! \equiv \lambda \quad(\bmod p)
$$

has a solvtion in positive integers $n_{1}, \ldots, n_{\ell}$.

THEOREM 1. For any prime $p, \ell(p)=O\left((\log p)^{3} \log \log p\right)$.

It certainly remains a challenging open question to prove that $\ell(p)=O(1)$, which we believe to be true.

\section{Preparations}

Here, we obtain some bounds on the number of solutions of some congruences and some exponential sums. We present them in forms more general that we need in the paper, but which we believe to be of independent interest.

The implied constants in symbols ' $O$ ' and ' $\ll$ ' are absolute (we recall that $U \ll V$ and $U=O(V)$ are both equivalent to the inequality $|U| \leqslant c V$ with some constant $c>0$ ). In particular, they do not depend on the auxiliary parameter $\ell$ which occurs in our estimates.

Received 25th October, 2004

During the preparation of this paper, the second author was supported in part by grants SEP-CONACYT 37259-E and 37260-E, and the third author was supported in part by ARC grant DP0211459.

Copyright Clearance Centre, Inc. Serial-fee code: 0004-9727/05 \$A2.00+0.00. 
We denote by $J_{\ell}(N)$ the number of solutions to the congruence

$$
\sum_{i=1}^{\ell} n_{i} ! \equiv \sum_{i=\ell+1}^{2 \ell} n_{i} ! \quad(\bmod p), \quad 1 \leqslant n_{1}, \ldots, n_{2 \ell} \leqslant N
$$

Our treatment of $J_{\ell}(N)$ is based on exponential sums. Accordingly, we define

$$
\mathbf{e}_{p}(z)=\exp (2 \pi i z / p)
$$

and recall the identity (see [9, Exercise 11.a in Chapter 3])

$$
\sum_{a=0}^{p-1} \mathbf{e}_{p}(a u)=\left\{\begin{array}{lll}
0, & \text { if } u \neq 00 & (\bmod p) \\
p, & \text { if } u \equiv 0 & (\bmod p)
\end{array}\right.
$$

which we repeatedly use, in particular to relate the number of solutions of various congruences and exponential sums.

Lemma 2. For any positive integers $\ell, N$ with $\ell<N<p$,

$$
J_{\ell}(N) \ll \ell^{2} N^{2 \ell-1+1 /(\ell+1)} .
$$

Proof: Let us define exponential sums

$$
S_{a}(N)=\sum_{n=1}^{N} \mathrm{e}_{p}(a n !)
$$

The identity (2) implies that

$$
J_{\ell}(N)=\frac{1}{p} \sum_{a=0}^{p-1}\left|S_{a}(N)\right|^{2 \ell}
$$

Put

$$
K=\left\lfloor\left(\frac{N^{\ell}}{\ell !}\right)^{1 /(\ell+1)}\right\rfloor
$$

Using the Hölder inequality, we derive

$$
\begin{aligned}
\left|S_{a}(p)\right|^{2 \ell} & =\left|\sum_{k=1}^{K} \sum_{(k-1) N K^{-1}<m \leqslant k N K^{-1}} \mathbf{e}_{p}(a n !)\right|^{2 \ell} \\
& \leqslant\left.\left. K^{2 k-1} \sum_{k=1}^{K}\right|_{(k-1) N K^{-1}<m \leqslant k N K^{-1}} \sum_{p}(a n !)\right|^{2 \ell}
\end{aligned}
$$

hence

$$
J_{\ell}(N) \leqslant K^{2 \ell-1} G_{\ell}(K, N)
$$


where $G_{\ell}(K, N)$ is the number of solutions of the congruence

$$
\sum_{i=1}^{\ell} m_{i} ! \equiv \sum_{i=\ell+1}^{2 \ell} m_{i} ! \quad(\bmod p), \quad 1 \leqslant m_{1}, \ldots, m_{2 \ell} \leqslant N,
$$

subject to the conditions $\left|m_{i}-m_{j}\right|<N K^{-1}$ for $1 \leqslant i, j \leqslant 2 \ell$.

Obviously, $G_{\ell}(K, N) \leqslant 2 \ell \widetilde{G}_{\ell}(K, N)$, where $\widetilde{G}_{\ell}(K, N)$ is number of solutions of the same congruence with the additional restriction that

$$
m_{1}=\min _{1 \leqslant i \leqslant 2 \ell} m_{i}
$$

To estimate $\widetilde{G}_{\ell}(K, N)$, we write $m_{i}=m+t_{i}$, where $t_{1}=0$ and $0 \leqslant t_{i}<N K^{-1}$, $2 \leqslant i \leqslant 2 \ell$. Then, after dividing by $m_{1} ! \neq \equiv 0(\bmod p)$, the above congruence takes the form

$$
F_{t_{1}, \ldots, t_{2 \ell}}(m) \equiv 0 \quad(\bmod p)
$$

where

$$
F_{t_{1}, \ldots, t_{2 \ell}}(X)=\sum_{i=1}^{\ell} \prod_{\nu=1}^{t_{i}}(X+\nu)-\sum_{i=\ell+1}^{2 \ell} \prod_{\nu=1}^{t_{i}}(X+\nu) .
$$

The polynomial $F_{t_{1}, \ldots, t_{2 \ell}}(X)$ vanishes modulo $p$ if and only if the sequence $t_{\ell+1}, \ldots, t_{2 \ell}$ is a permutation of the sequence $t_{1}=0, t_{2}, . ., t_{\ell}$. Therefore, this happens for at most $\ell !\left(N K^{-1}+1\right)^{\ell-1}$ values of $t_{1}=0, t_{2}, . ., t_{2 \ell}$. For these values, the congruence (4) is satisfied for all values of $m=1, \ldots, N$. For the other at most $\left(N K^{-1}+1\right)^{2 \ell-1}$ values of $t_{1}=0, t_{2}, . ., t_{2 \ell}$, the congruence (4) is satisfied for at most $\operatorname{deg} F_{t_{1}, \ldots, t_{2 \ell}} \leqslant N K^{-1}+1$ values of $m$. Hence,

$$
\begin{aligned}
\tilde{G}_{\ell}(K, N) & \leqslant \ell !\left(N K^{-1}+1\right)^{\ell-1} N+\left(N K^{-1}+1\right)^{2 \ell} \\
& =\ell ! N^{\ell} K^{-\ell+1}\left(1+K N^{-1}\right)^{\ell-1}+N^{2 \ell} K^{-2 \ell}\left(1+K N^{-1}\right)^{2 \ell} .
\end{aligned}
$$

We now remark that by our choice of $K$ and the Stirling formula, we have

$$
1+K N^{-1} \leqslant 1+\left(\frac{N^{-1}}{\ell !}\right)^{1 /(\ell+1)}=1+O(1 / \ell) .
$$

Therefore,

$$
\widetilde{G}_{\ell}(K, N) \ll \ell ! N^{\ell} K^{-\ell+1}+N^{2 \ell} K^{-2 \ell},
$$

and hence,

$$
J_{\ell}(N) \ll \ell\left(\ell ! N^{\ell} K^{\ell}+N^{2 \ell} K^{-1}\right) .
$$

By our choice of $K$, we see that

$$
\ell ! N^{\ell} K^{\ell} \leqslant N^{2 \ell} K^{-1}
$$


and also that for sufficiently large $N$

$$
K \geqslant\left(\frac{N^{\ell}}{\ell !}\right)^{1 /(\ell+1)}-1 \geqslant \frac{N^{\ell /(\ell+1)}}{2(\ell !)^{1 /(\ell+1)}} .
$$

Therefore,

$$
J_{\ell}(N) \ll \ell(\ell !)^{1 /(\ell+1)} N^{2 \ell-1+1 /(\ell+1)},
$$

which finishes the proof.

For positive integers $k, H, N<p$ we now consider double exponential sums of the form

$$
W_{a}(k ; H, N)=\sum_{h=1}^{H}\left|\sum_{n=1}^{N} \mathrm{e}_{p}(a h n !)\right|^{k} .
$$

We estimate $W_{a}(k ; H, N)$ using essentially the same arguments as in $[9$, Exercise 14.a in Chapter VI].

LEMma 3. For any integer $\ell \geqslant 1$,

$$
\left|W_{a}(k ; H, N)\right| \leqslant\left(p H J_{k}(N)\right)^{1 / 2} .
$$

Proof: Using the Hölder inequality we derive

$$
\begin{aligned}
\left|W_{a}(k ; H, p)\right|^{2} & \leqslant H \sum_{h=1}^{H}\left|\sum_{n=1}^{N} \mathrm{e}_{p}(a h n !)\right|^{2 k} \\
& \leqslant H \sum_{h=1}^{p}\left|\sum_{n=1}^{N} \mathrm{e}_{p}(a h n !)\right|^{2 k}=p H J_{k}(N),
\end{aligned}
$$

which finishes the proof.

\section{Proof of Theorem 1}

For some positive integers $s, \ell, H<p$ we denote by $T$ the number of solutions of the congruence

$$
\sum_{i=1}^{2 s} h_{i}\left(n_{i 1} !+\cdots+n_{i \ell} !\right)+\sum_{i=1}^{2 \ell} m_{i} ! \equiv \lambda \quad(\bmod p),
$$

where

$$
1 \leqslant n_{1,1}, \ldots, n_{2 s, \ell}, m_{1}, \ldots, m_{2 \ell} \leqslant p-1, \quad 1 \leqslant h_{1}, \ldots, h_{2 s} \leqslant H .
$$

By the identity (2), we have

$$
T=\frac{1}{p} \sum_{a=0}^{p-1}\left(\sum_{h=1}^{H}\left(\sum_{n=1}^{p} \mathrm{e}_{p}(a h n !)\right)^{\ell}\right)^{2 s}\left(\sum_{m=1}^{p} \mathrm{e}_{p}(a m !)\right)^{2 \ell} .
$$


Separating the term $H^{2 s} p^{2 s \ell+2 \ell-1}$ corresponding to $a=0$, we derive

$$
\left|T-H^{2 s} p^{2 s \ell+2 \ell-1}\right| \leqslant \frac{1}{p} \sum_{a=1}^{p-1}\left(\sum_{h=1}^{H}\left|\sum_{n=1}^{p} \mathrm{e}_{p}(a h n !)\right|^{\ell}\right)^{2 s}\left|\sum_{m=1}^{p} \mathrm{e}_{p}(a m !)\right|^{2 \ell} .
$$

Thus, using Lemma 3 and then Lemma 2, we get the inequalities

$$
\begin{aligned}
\left|T-H^{2 s} p^{2 s \ell+2 \ell-1}\right| & \leqslant \frac{1}{p}\left(p H J_{\ell}(p)\right)^{s} \sum_{a=1}^{p-1}\left|\sum_{m=1}^{p} \mathrm{e}_{p}(a n !)\right|^{2 s} \\
& \leqslant p^{s} H^{s}\left(J_{\ell}(p)\right)^{s+1} \ll H^{s} \ell^{2 s+2} p^{2 \ell+2 \ell-1+(s+1) /(\ell+1)} .
\end{aligned}
$$

Therefore, the inequality

$$
T \geqslant H^{2 s} p^{2 s \ell+2 \ell-1}-C H^{s} \ell^{2 s+2} p^{2 \ell s+2 \ell-1+(s+1) /(\ell+1)}
$$

holds with some absolute constant $C>0$. Selecting $\ell=\lfloor\log p\rfloor, s=\lfloor\log \log p\rfloor$, and $H=\left\lceil e^{5} \ell^{2}\right\rceil$, we see that $T>0$. Adding, if necessary, several terms of the form $p$ !, we see that every residue class $\lambda$ modulo $p$ can be represented by a sum of the same number $\ell(p)$ of factorials, where $\ell(p) \leqslant 2 s \ell H+2 \ell \ll(\log p)^{3} \log \log p$.

\section{REMARKS}

As we have mentioned, the question whether $\ell(p)=O(1)$ remains open. In $[4, F 11]$, it is conjectured that the values of $n$ ! hit about $(1-1 / e) p$ of the residue classes modulo $p$. Proving such a result would immediately imply that $\ell(p)=2$. Unfortunately this conjecture appears to be very hard.

Our method can also be used, without any substantial changes, to study the Waring problem with more general functions $F(n)$ given by the products

$$
F(n)=\prod_{j=1}^{n} f(j) \quad(\bmod p)
$$

where $f(j)$ is any rational function defined modulo $p$ which has no zeros or poles for all $j=1, \ldots, p$. In particular, one can easily extend our results to congruences

$$
\left(n_{1} !\right)^{k}+\cdots+\left(n_{\ell} !\right)^{k} \equiv \lambda \quad(\bmod p)
$$

with a fixed integer $k \geqslant 1$. In fact one can also consider negative values of $k$.

\section{REFERENCES}

[1] M.Z. Garaev and F. Luca, 'Character sums and products of factorials modulo $p$ ', $J$. Théor. Nombres Bordeaux (to appear). 
[2] M.Z. Garaev, F. Luca and I.E. Shparlinski, 'Character sums and congruences with n!', Trans. Amer. Math. Soc. 356 (2004), 5089-5102.

[3] M.Z. Garaev, F. Luca and I.E. Shparlinski, 'Exponential sums and congruences with factorials', J. Reine Angew. Math. (to appear).

[4] R.K. Guy, Unsolved problems in number theory (Springer-Verlag, New York, 1994).

[5] F. Luca and I.E. Shparlinski, 'Prime divisors of shifted factorials', Bull. Lond. Math. Soc. (to appear).

[6] F. Luca and I.E. Shparlinski, 'On the largest prime factor of $n !+2^{n}-1$ ', J. Théor. Nombres Bordeaux (to appear).

[7] F. Luca and P. Stănică, 'Products of factorials modulo p', Colloq. Math. 96 (2003), 191-205.

[8] C. Stewart, 'On the greatest and least prime factors of $n !+1, \mathrm{II}$ ', Publ. Math. Debrecen 65 (2004), 461-480.

[9] I.M. Vinogradov, Elements of number theory (Dover Publications, New York, 1954).

Instituto de Matemáticas

Universidad Nacional Autónoma de México

C.P. 58180, Morelia

Michoacán

México

e-mail: garaev@matmor.unam.mx

fluca@matmor.unam.mx
Department of Computing

Macquarie University

Sydney, NSW 2109

Australia

e-mail: igor@ics.mq.edu.au 